\title{
On topological Rudin's lemma, well-filtered spaces and sober spaces ${ }^{\text {th }}$
}

\author{
Xiaoquan $\mathrm{Xu}^{\mathrm{a}, *}$, Dongsheng Zhao ${ }^{\mathrm{b}}$ \\ ${ }^{a}$ School of Mathematics and Statistics, Minnan Normal University, Zhangzhou 363000, China \\ ${ }^{b}$ Mathematics and Mathematics Education, National Institute of Education Singapore, \\ Nanyang Technological University, 1 Nanyang Walk, Singapore 637616
}

\begin{abstract}
Based on topological Rudin's Lemma, we investigate two new kinds of sets - Rudin sets and well-filtered determined sets in $T_{0}$ topological spaces. Using such sets, we formulate and prove some new characterizations for well-filtered spaces and sober spaces. Part of the work was inspired by Xi and Lawson's work on wellfiltered spaces. Our study also lead to a new class of spaces - strong $d$-spaces and some problems whose solutions will strengthen our understanding of the related structures.
\end{abstract}

Keywords: Topological Rudin's lemma; Sober space; Well-filtered space; $d$-space; Strong $d$-space 2000 MSC: 06B35; 06F30; 54B99; 54D30

In domain theory, the $d$-spaces, well-filtered spaces and sober spaces form three of the most important classes of spaces. Rudin's Lemma has played a crucial role in studying such spaces. The original application of Rudin's Lemma was in answering some questions on quasicontinuous dcpos. In recent years, it has been used to study the various aspects of well-filtered spaces, initiated by Heckmann and Keimel [7]. In this paper, inspired by the topological version of Rudin's Lemma by Heckmann and Keimel, Xi and Lawson's work [16] on well-filtered spaces and our recent work [14] on the well-filtered reflections of $T_{0}$ spaces, we investigate two new kinds of sets - Rudin sets and well-filtered determined sets in $T_{0}$ topological spaces, and use them to establish a series new characterizations of well-filtered spaces and sober spaces. Our study also leads to a new class of spaces - strong $d$-spaces, and a number of problems, whose answering will deepen our understanding of the related spaces and structures.

\section{Preliminary}

For a poset $P$ and $A \subseteq P$, let $\downarrow A=\{x \in P: x \leq a$ for some $a \in A\}$ and $\uparrow A=\{x \in P: x \geq$ $a$ for some $a \in A\}$. For $x \in P$, we write $\downarrow x$ for $\downarrow\{x\}$ and $\uparrow x$ for $\uparrow\{x\}$. A subset $A$ is called a lower set (resp., an upper set) if $A=\downarrow A$ (resp., $A=\uparrow A$ ). Let $P^{(<\omega)}=\{F \subseteq P: F$ is a nonempty finite set $\}$, $P^{(\leqslant \omega)}=\{F \subseteq P: F$ is a nonempty countabel set $\}$ and Fin $P=\left\{\uparrow F: F \in P^{(<\omega)}\right\}$. The set of all nonempty upper subsets of $P$ is denoted by $\mathbf{u p}(P)$. For a nonempty subset $B$ of $P$, let $\max (B)=\{b \in B$ : $b$ is a maximal element of $B\}$ and $\min (B)=\{b \in B: b$ is a minimal element of $B\}$. A nonempty subset $D$ of $P$ is directed if every two elements in $D$ have an upper bound in $D$. The set of all directed sets of $P$ is denoted by $\mathcal{D}(P)$. A subset $I \subseteq P$ is called an ideal of $P$ if $I$ is a directed and a lower set. $\operatorname{Let} \operatorname{Id}(P)$ be the poset (with the order of set inclusion) of all ideals of $P$. Dually, we define the concept of filters and denote the poset of all filters of $P$ by Filt $(P)$. $P$ is called a directed complete poset, or dcpo for short, if for any $D \in \mathcal{D}(P), \bigvee D$ exists in $P$.

As in 4, the lower topology on a poset $P$, generated by the complements of the principal filters of $P$, is denoted by $\omega(P)$. Dually, define the upper topology on $P$ and denote it by $v(P)$. A subset $U$ of $P$ is $S c o t t$

\footnotetext{
This research was supported by the National Natural Science Foundation of China (No. 11661057); the Natural Science Foundation of Jiangxi Province, China (No. 20192ACBL20045); and NIE ACRF (RI 3/16 ZDS), Singapore 
open if (i) $U=\uparrow U$ and (ii) for any directed subset $D$ for which $\bigvee D$ exists, $\bigvee D \in U$ implies $D \cap U \neq \emptyset$. All Scott open subsets of $P$ form a topology, and we call this topology the Scott topology on $P$ and denote it by $\sigma(P)$. The space $\Sigma P=(P, \sigma(P))$ is called the $S$ cott space of $P$. The common refinement $\sigma(P)$ and $\omega(P)$ is called the Lawson topology and is denoted by $\lambda(P)$. The upper sets form the (upper) Alexandroff topology $\alpha(P)$.

A poset $Q$ is called an (inf) semilattice if for any two elements $a, b \in Q, \inf \{a, b\}=a \wedge b$ exists in $Q$. Dually, $Q$ is a sup semilattice if for any two elements $a, b \in Q, \sup \{a, b\}=a \vee b$ exists in $Q$.

Definition 1.1. Let $P$ be a poset.

(i) $P$ is called a complete semilattice if $P$ is a dcpo and every nonempty subset $P$ has an inf. In particular, a complete semilattice has a smallest element, the inf of $P$.

(ii) $P$ is called bounded complete if every subset that is bounded above has a sup (i.e., the least upper bound). In particular, a bounded complete poset has a smallest element, the least upper bound of the empty set.

It is easy to see that $P$ is a complete semilattice iff $P$ is a bounded complete dcpo (see, e,g., 4, Proposition $\mathrm{O}-2.2])$.

For a $T_{0}$ space $X$, we use $\leq_{X}$ to represent the specialization order of $X$, that is, $x \leq_{X} y$ iff $x \in \overline{\{y\}}$. In the following, when a $T_{0}$ space $X$ is considered as a poset, the order always refers to the specialization order if no other explanation. Let $\mathcal{O}(X)$ (resp., $\mathcal{C}(X)$ ) be the set of all open subsets (resp., closed subsets) of $X$, and let $\mathcal{S}^{u}(X)=\{\uparrow x: x \in X\}, \mathcal{S}_{c}(X)=\{\overline{\{x\}}: x \in X\}$ and $\mathcal{D}_{c}(X)=\{\bar{D}: D \in \mathcal{D}(X)\}$. A space $X$ is called a $d$-space (or monotone convergence space) if $X$ (endowed with the specialization order) is a dcpo and $\mathcal{O}(X) \subseteq \sigma(X)$ (cf. [4][15]).

Proposition 1.2. For a $T_{0}$ space $X$, the following conditions are equivalent:

1. $X$ is a d-space.

2. $\mathcal{D}_{c}(X)=\mathcal{S}_{c}(X)$.

3. For any $D \in \mathcal{D}(X)$ and $U \in \mathcal{O}(X), \bigcap_{d \in D} \uparrow d \subseteq U$ implies $\uparrow d \subseteq U$ (i.e., $d \in U$ ) for some $d \in D$.

4. For any $D \in \mathcal{D}(X)$ and $A \in \mathcal{C}(X)$, if $D \subseteq A$, then $A \cap \bigcap_{d \in D} \uparrow d \neq \emptyset$.

5. For any $D \in \mathcal{D}(X), \bar{D} \cap \bigcap_{d \in D} \uparrow d \neq \emptyset$.

Proof. (1) $\Leftrightarrow(2)$ : If $X$ is a $d$-space, then for any $D \in \mathcal{D}(X), \bar{D}=\overline{\{}$ sup $D\}$, thus $(1) \Rightarrow(2)$. Conversely, if condition (2) holds, then for each $D \in \mathcal{D}(X)$ and $A \in \mathcal{C}(X)$ with $D \subseteq A$, there is $x \in X$ such that $\bar{D}=\overline{\{x\}}$, and consequently, $\bigvee D=x$ and $\bigvee D \in A$ since $\bar{D} \subseteq A$. Thus $X$ is a dcpo and $\mathcal{O}(X) \subseteq \sigma(X)$, hence $X$ is a $d$-space.

$(1) \Rightarrow(3)$ : Since $X$ is a $d$-space, $\uparrow \bigvee D=\bigcap_{d \in D} \uparrow d \subseteq U \in \sigma(X)$. Therefore, $\bigvee D \in U$, thus $d \in U$ for some $d \in D$.

(3) $\Rightarrow$ (4): If $A \cap \bigcap_{d \in D} \uparrow d=\emptyset$, then $\bigcap_{d \in D} \uparrow d \subseteq X \backslash A$. By condition (3), $\uparrow d \subseteq X \backslash A$ for some $d \in D$, which contradicts $D \subseteq A$.

$(4) \Rightarrow(5)$ : Trivial.

$(5) \Rightarrow(1)$ : For each $D \in \mathcal{D}(X)$ and $A \in \mathcal{C}(X)$ with $D \subseteq A$, by condition (5), $\bar{D} \cap \bigcap_{d \in D} \uparrow d \neq \emptyset$. Choose one $x \in \bar{D} \cap \bigcap_{d \in D} \uparrow d$. Then $D \subseteq \downarrow x \subseteq \bar{D}$, hence $\bar{D}=\downarrow x$ and $\bigvee D=x$. Therefore, $\bigvee D \in A$ because $\overline{\{\bigvee D\}}=\bar{D} \subseteq A$. Thus $X$ is a $d$-space.

Lemma 1.3. If $X$ is a $d$-space and $A$ is a nonempty closed subset of $X$, then $\max (A) \neq \emptyset$.

Proof. By Zorn's Lemma there is a maximal chain $C$ in $A$. Since $X$ is a $d$-space, $c=\bigvee C$ exists and $c \in A$. By the maximality of $C$, we have $c \in \max (A)$.

A nonempty subset $A$ of a $T_{0}$ space $X$ is said to be irreducible if for any $\left\{F_{1}, F_{2}\right\} \subseteq \mathcal{C}(X), A \subseteq F_{1} \cup F_{2}$ implies $A \subseteq F_{1}$ or $A \subseteq F_{2}$. Denote by $\operatorname{Irr}(X)$ (resp., $\operatorname{Irr}_{c}(X)$ ) the set of all irreducible (resp., irreducible closed) subsets of $X$. Every subset of $X$ that is directed under $\leq_{X}$ is irreducible. A space $X$ is called sober, if for any $F \in \operatorname{Irr}_{c}(X)$, there is a unique point $a \in X$ such that $F=\overline{\{a\}}$.

The following two lemmas on irreducible sets are well-known that will be used in the sequel. 
Lemma 1.4. Let $X$ be a space and $Y$ a subspace of $X$. Then the following conditions are equivalent for a subset $A \subseteq Y$ :

(1) $A$ is an irreducible subset of $Y$.

(2) $A$ is an irreducible subset of $X$.

(3) $\operatorname{cl}_{X} A$ is an irreducible subset of $X$.

Lemma 1.5. If $f: X \longrightarrow Y$ is continuous and $A \in \operatorname{Irr}(X)$, then $f(A) \in \operatorname{Irr}(Y)$.

Remark 1.6. If $Y$ is a subspace of a space $X$ and $A \subseteq Y$, then by Lemma 1.4 $\operatorname{Irr}(Y)=\{B \in \operatorname{Irr}(X): B \subseteq$ $Y\} \subseteq \operatorname{Irr}(X)$ and $\operatorname{Irr}_{c}(Y)=\{B \in \operatorname{Irr}(X): B \in \mathcal{C}(Y)\} \subseteq \operatorname{Irr}(X)$. If $Y \in \mathcal{C}(X)$, then $\operatorname{Irr}_{c}(Y)=\left\{C \in \operatorname{Irr}_{c}(X)\right.$ : $C \subseteq Y\} \subseteq \operatorname{Irr}_{c}(X)$.

For any topological space $X, \mathcal{G} \subseteq 2^{X}$ and $A \subseteq X$, let $\diamond_{\mathcal{G}} A=\{G \in \mathcal{G}: G \bigcap A \neq \emptyset\}$ and $\square_{\mathcal{G}} A=\{G \in \mathcal{G}$ : $G \subseteq A\}$. The sets $\diamond_{\mathcal{G}} A$ and $\square_{\mathcal{G}} A$ will be simply written as $\diamond A$ and $\square A$, respectively, if there is no confusion. The upper Vietoris topology on $\mathcal{G}$ is the topology that has $\left\{\square_{\mathcal{G}} U: U \in \mathcal{O}(X)\right\}$ as a base and the resulting space is denoted by $P_{S}(\mathcal{G})$. The lower Vietoris topology on $\mathcal{G}$ is the topology that has $\{\diamond U: U \in \mathcal{O}(X)\}$ as a subbase and the resulting space is denoted by $P_{H}(\mathcal{G})$. If $\mathcal{G} \subseteq \operatorname{Irr}(X)$, then $\left\{\diamond_{\mathcal{G}} U: U \in \mathcal{O}(X)\right\}$ is a topology on $\mathcal{G}$. The space $P_{H}(\mathcal{C}(X) \backslash\{\emptyset\})$ is called the Hoare power space or lower space of $X$ and is denoted by $P_{H}(X)$ for short (cf. [13]). Clearly, $P_{H}(X)=\left(\mathcal{C}(X) \backslash\{\emptyset\}, v(\mathcal{C}(X) \backslash\{\emptyset\})\right.$ ). So $P_{H}(X)$ is always sober (see, e.g., [17, Corollary 4.10]). The space $P_{H}\left(\operatorname{Irr}_{c}(X)\right)$, shortly denoted by $X^{s}$, with the topological embedding $\eta_{X}(=x \mapsto \overline{\{x\}}): X \longrightarrow P_{H}\left(\operatorname{Irr}_{c}(X)\right)$, is the canonical soberification of $X$ (cf. [4).

A subset $A$ of a space $X$ is called saturated if $A$ equals the intersection of all open sets containing it (equivalently, $A$ is an upper set with respect to the specialization order). We shall use $\mathrm{K}(X)$ to denote the set of all nonempty compact saturated subsets of $X . X$ is called coherent if the intersection of any two compact saturated sets is again compact. $X$ is said to be well-filtered if it is $T_{0}$, and for any open set $U$ and filtered family $\mathcal{K} \subseteq \mathrm{K}(X), \bigcap \mathcal{K} \subseteq U$ implies $K \subseteq U$ for some $K \in \mathcal{K}$. The space $P_{S}(\mathrm{~K}(X))$, denoted shortly by $P_{S}(X)$, is called the Smyth power space or upper space of $X$ (cf. [6] 13]). The space $P_{S}(\mathbf{u p}(X))$ is called the Alexandroff power space. It is easy to see that $P_{S}(X)$ is a subspace of $P_{S}(\mathbf{u p}(X))$, and the specialization orders on $P_{S}(\mathbf{u p}(X))$ is the Smyth preorder, that is, for $K_{1}, K_{2} \in \mathbf{u p}(X), K_{1} \leq_{P_{S}(\mathbf{u p}(X))} K_{2}$ iff $K_{2} \subseteq K_{1}$. The canonical mapping $\xi_{X}: X \longrightarrow P_{S}(X), x \mapsto \uparrow x$, is an order and topological embedding (cf. [6] [7] [13]). Clearly, $P_{S}\left(\mathcal{S}^{u}(X)\right)$ is a subspace of $P_{S}(X)$ and $X$ is homeomorphic to $P_{S}\left(\mathcal{S}^{u}(X)\right)$.

By Lemma 1.4 and Lemma 1.5. we have the following corollary.

Corollary 1.7. Let $X$ be a $T_{0}$ space and $\mathcal{A} \subseteq \mathrm{K}(X)$. Then the following three conditions are equivalent:

(1) $\mathcal{A} \in \operatorname{Irr}\left(P_{S}(X)\right)$

(2) $\mathcal{A} \in \operatorname{Irr}\left(P_{S}(\mathbf{u p}(X))\right)$.

(3) $\operatorname{cl}_{P_{S}(\mathbf{u p}(X))} \mathcal{A} \in \operatorname{Irr}_{c}\left(P_{S}(\mathbf{u p}(X))\right)$.

Remark 1.8. Let $X$ be a $T_{0}$ space and $\mathcal{A} \subseteq \mathrm{K}(X)$ (resp., $\mathcal{A} \subseteq \mathbf{u p}(X)$ ). Then $\bigcap \mathcal{A}=\bigcap \overline{\mathcal{A}}$, here the closure of $\mathcal{A}$ is taken in $P_{S}(X)$ (resp., in $P_{S}(\mathbf{u p}(X))$ ). Clearly, $\cap \overline{\mathcal{A}} \subseteq \bigcap \mathcal{A}$. On the other hand, for $K \in \overline{\mathcal{A}}$ and $U \in \mathcal{O}(X)$ with $K \subseteq U$ (that is, $K \in \square U)$, we have $\mathcal{A} \cap \square U \neq \emptyset$, and hence there is a $K_{U} \in \mathcal{A} \cap \square U$. Therefore $K=\bigcap\{U \in \mathcal{O}(X): K \subseteq U\} \supseteq \bigcap\left\{K_{U}: U \in \mathcal{O}(X)\right.$ and $\left.K \subseteq U\right\} \supseteq \bigcap \mathcal{A}$. It follows that $\bigcap \overline{\mathcal{A}} \supseteq \bigcap \mathcal{A}$. Thus $\bigcap \mathcal{A}=\bigcap \overline{\mathcal{A}}$.

\section{Rudin sets and well-filtered determined sets}

Rudin's Lemma plays a crucial role in domain theory (see [1-7]). Rudin [12 proved her lemma by transfinite methods. Later, Heckmann and Keimel [7] established the following topological variant of Rudin's Lemma.

Lemma 2.1. (Topological Rudin's Lemma) Let $X$ be a topological space and $\mathcal{A}$ an irreducible subset of the Smyth power space $P_{S}(X)$. Then every closed set $C \subseteq X$ that meets all members of $\mathcal{A}$ contains an minimal irreducible closed subset $A$ that meets all members of $\mathcal{A}$. 
Applying Lemma 2.1 to the Alexandroff topology on a poset $P$, one obtains the original Rudin's Lemma.

Corollary 2.2. (Rudin's Lemma) Let $P$ be a poset, $C$ a nonempty lower subset of $P$ and $\mathcal{F} \in \mathbf{F i n} P$ a filtered family with $\mathcal{F} \subseteq \diamond C$. Then there exists a directed subset $D$ of $C$ such that $\mathcal{F} \subseteq \diamond \downarrow D$.

For a $T_{0}$ space $X$ and $\mathcal{K} \subseteq \mathrm{K}(X)$, let $M(\mathcal{K})=\{A \in \mathcal{C}(X): K \bigcap A \neq \emptyset$ for all $K \in \mathcal{K}\}$ (that is, $\left.\mathcal{A} \subseteq \diamond A\right)$ and $m(\mathcal{K})=\{A \in \mathcal{C}(X): A$ is a minimal menber of $M(\mathcal{K})\}$.

Definition 2.3. ([14]) Let $X$ be a $T_{0}$ space. A nonempty subset $A$ of $X$ is said to have the Rudin property, if there exists a filtered family $\mathcal{K} \subseteq \mathrm{K}(X)$ such that $\bar{A} \in m(\mathcal{K})$ (that is, $\bar{A}$ is a minimal closed set that intersects all members of $\mathcal{K})$. Let $\operatorname{RD}(X)=\{A \in \mathcal{C}(X): A$ has Rudin property $\}$.

The sets in $\mathrm{RD}(X)$ will also be called Rudin sets.

The Rudin property is called the compactly filtered property in [14. In order to emphasize its origin, here we call such property the Rudin property.

Proposition 2.4. Let $X$ be a $T_{0}$ space and $Y$ a well-filtered space. If $f: X \longrightarrow Y$ is continuous and $A \subseteq X$ is a Rudin set, then there exists a unique $y_{A} \in X$ such that $\overline{f(A)}=\overline{\left\{y_{A}\right\}}$.

Proof. Since $A$ has Rudin property, there exists a filtered family $\mathcal{K} \subseteq \mathrm{K}(X)$ such that $\bar{A} \in m(\mathcal{K})$. Let $\mathcal{K}_{f}=\{\uparrow f(K \cap \bar{A}): K \in \mathcal{K}\}$. Then $\mathcal{F}_{f} \subseteq \mathrm{K}(Y)$ is filtered. By the proof of Lemma 2.7 $\overline{f(A)} \in m\left(\mathcal{K}_{f}\right)$. By the well-filteredness of $Y, \bigcap_{K \in \mathcal{K}} \uparrow f(K \cap \bar{A}) \cap \overline{f(A)} \neq \emptyset$. Select a $y_{A} \in \bigcap_{K \in \mathcal{K}} \uparrow f(K \cap \bar{A}) \cap \overline{f(A)}$, then $\overline{\left\{y_{A}\right\}} \subseteq \overline{f(A)}$ and $K \cap \bar{A} \cap \underline{f^{-1}}\left(\overline{\left\{y_{A}\right\}}\right) \neq \emptyset$ for all $K \in \mathcal{K}$. It follows that $\bar{A}=\bar{A} \cap f^{-1}\left(\overline{\left\{y_{A}\right\}}\right)$ by the minimality of $\bar{A}$, and consequently, $\overline{f(A)} \subseteq \overline{\left\{y_{A}\right\}}$. Therefore, $\overline{f(A)}=\overline{\left\{y_{A}\right\}}$. The uniqueness of $y_{A}$ follows from the $T_{0}$ separation of $Y$.

Motivated by Proposition 2.4, we give the following definition.

Definition 2.5. A subset $A$ of a space $X$ is called a well-filtered determined set if for any continuous mapping $f: X \longrightarrow Y$ into a well-filtered space $Y$, there exists a unique $y_{A} \in Y$ such that $\overline{f(A)}=\overline{\left\{y_{A}\right\}}$. Denote by $\operatorname{WD}(X)$ the set of all closed well-filtered determined subsets of $X$.

Obviously, a subset $A$ of a space $X$ is well-filtered determined iff $\bar{A}$ is well-filtered determined.

Proposition 2.6. Let $X$ be a $T_{0}$ space. Then $S_{c}(X) \subseteq \mathcal{D}_{c}(X) \subseteq \mathrm{RD}(X) \subseteq \mathrm{WD}(X) \subseteq \operatorname{Irr}_{c}(X)$.

Proof. Obviously, $S_{c}(X) \subseteq \mathcal{D}_{c}(X)$. Now we prove that the closure of a directed subset $D$ of $X$ is a Rudin set. Let $\mathcal{K}_{D}=\{\uparrow d: d \in D\}$. Then $\mathcal{K}_{D} \subseteq \mathrm{K}(X)$ is filtered and $\bar{D} \in M\left(\mathcal{K}_{D}\right)$. If $A \in M\left(\mathcal{K}_{D}\right)$, then $d \in A$ for every $d \in D$, hence $\bar{D} \subseteq A$. So $\bar{D} \in m\left(\mathcal{K}_{D}\right)$. Therefore $\bar{D} \in \operatorname{RD}(X)$. By Proposition 2.4. we have $\mathrm{RD}(X) \subseteq \mathrm{WD}(X)$. Finally, $\overline{\mathrm{We}}$ show $\mathrm{WD}(X) \subseteq \operatorname{Irr}_{c}(X)$. Let $A \in \mathrm{WD}(X)$. Since $\eta_{X}: X \longrightarrow X^{s}, x \mapsto \downarrow x$, is a continuous mapping into a well-filtered space $\left(X^{s}\right.$ is sober), there exists $C \in \operatorname{Irr}_{c}(X)$ such that $\overline{\eta_{X}(A)}=$ $\overline{\{C\}}$. Let $U \in \mathcal{O}(X)$. Note that

$$
\begin{aligned}
A \cap U \neq \emptyset & \Leftrightarrow \eta_{X}(A) \cap \diamond U \neq \emptyset \\
& \Leftrightarrow\{C\} \cap \diamond U \neq \emptyset \\
& \Leftrightarrow C \in \diamond U \\
& \Leftrightarrow C \cap U \neq \emptyset .
\end{aligned}
$$

This implies that $A=C$, so $A \in \operatorname{Irr}_{c}(X)$.

Lemma 2.7. Let $X, Y$ be two $T_{0}$ spaces and $f: X \longrightarrow Y$ a continuous mapping.

(1) If $A \in \mathrm{RD}(X)$, then $\overline{f(A)} \in \mathrm{RD}(Y)$.

(2) If $A \in \mathrm{WD}(X)$, then $\overline{f(A)} \in \mathrm{WD}(Y)$. 
Proof. (1): It has been proved in [14. Here we give a more direct proof. Since $A \in \operatorname{RD}(X)$, there exists a filtered family $\mathcal{K} \subseteq \mathrm{K}(X)$ such that $A \in m(\mathcal{K})$. Let $\mathcal{K}_{f}=\{\uparrow f(K \cap A): K \in \mathcal{K}\}$. Then $\mathcal{K}_{f} \subseteq \mathrm{K}(Y)$ is filtered. For each $K \in \mathcal{K}$, since $K \cap A \neq \emptyset$, we have $\emptyset \neq f(K \cap A) \subseteq \uparrow f(K \cap A) \cap \overline{f(A)}$. So $\overline{f(A)} \in M\left(\mathcal{K}_{f}\right)$. If $B$ is a closed subset of $\overline{f(A)}$ with $B \in M\left(\mathcal{K}_{f}\right)$, then $B \cap \uparrow f(K \cap A) \neq \emptyset$ for every $K \in \mathcal{K}$. So $K \cap A \cap f^{-1}(B) \neq \emptyset$ for $\underline{\text { all } K} \in \mathcal{K}$. It follows that $A=A \cap f^{-1}(B)$ by the minimality of $A$, and consequently, $\overline{f(A)} \subseteq B$. Therefore, $\overline{f(A)}=B$. Thus $\overline{f(A)} \in \mathrm{RD}(Y)$.

(2): Let $Z$ be a well-filtered space and $g: Y \longrightarrow Z$ a continuous mapping. Since $g \circ f: X \longrightarrow Z$ is continuous and $A \in \mathrm{WD}(X)$, there is $z \in Z$ such that $\overline{g(\overline{f(A)})}=\overline{g \circ f(A)}=\overline{\{z\}}$. Thus $\overline{f(A)} \in \mathrm{WD}(Y)$.

Lemma 2.8. ([2]) Let $X$ be a locally hypercompact $T_{0}$ space and $A \in \operatorname{Irr}(X)$. Then there exists a directed subset $D \subseteq \downarrow A$ such that $\bar{A}=\bar{D}$.

Corollary 2.9. For any locally hypercompact $T_{0}$ space $X, \operatorname{Irr}_{c}(X)=\mathrm{WD}(X)=\operatorname{RD}(X)=\mathcal{D}_{c}(X)$.

Proposition 2.10. For any locally compact $T_{0}$ space $X, \operatorname{Irr}_{c}(X)=\mathrm{WD}(X)=\operatorname{RD}(X)$.

Proof. Suppose that $A \in \operatorname{Irr}_{c}(X)$. Let $\mathcal{K}_{A}=\{K \in \mathrm{K}(X): A \cap \operatorname{int} K \neq \emptyset\}$.

Claim 1: $\mathcal{K}_{A} \neq \emptyset$.

Let $a \in A$. Since $X$ is locally compact, there exists $K \in \mathrm{K}(X)$ such that $a \in \operatorname{int} K$. So $a \in A \cap \operatorname{int} K$ and $K \in \mathcal{K}_{A}$, implying $\mathcal{K}_{A} \neq \emptyset$.

Claim 2: $\mathcal{K}_{A}$ is filtered.

Let $K_{1}, K_{2} \in \mathcal{K}_{A}$. Then $A \cap \operatorname{int} K_{1} \neq \emptyset$ and $A \cap \operatorname{int} K_{2} \neq \emptyset$. Since $A$ is irreducible, $A \cap \operatorname{int} K_{1} \cap \operatorname{int} K_{2} \neq \emptyset$. Let $x \in A \cap \operatorname{int} K_{1} \cap \operatorname{int} K_{2}$. By the local compactness of $X$ again, there exists $K_{3} \in \mathrm{K}(X)$ such that $x \in \operatorname{int} K_{3} \subseteq K_{3} \subseteq \operatorname{int} K_{1} \cap \operatorname{int} K_{2}$. Thus $K_{3} \in \mathcal{K}_{A}$ and $K_{3} \subseteq K_{1} \cap K_{2}$. So $\mathcal{K}_{A}$ is filtered.

Claim 3: $A \in m\left(\mathcal{K}_{A}\right)$.

Clearly, $\mathcal{K}_{A} \subseteq \diamond A$. If $B$ is a proper closed subset of $A$, then there is $a \in A \backslash B$. Since $X$ is locally compact, there is $K_{a} \in \mathrm{K}(X)$ such that $a \in \operatorname{int} K_{a} \subseteq K_{a} \subseteq X \backslash B$. Then $K_{a} \in \mathcal{K}_{A}$ but $K_{a} \cap B=\emptyset$. Thus $A$ is a minimal closed set that meets all members of $\mathcal{K}_{A}$, and hence $A \in \operatorname{RD}(X)$. By Proposition 2.6. $\operatorname{Irr}_{c}(X)=\mathrm{WD}(X)=\mathrm{RD}(X)$.

\section{Some new characterizations of well-filtered spaces and sober spaces}

In [16, $\mathrm{Xi}$ and Lawson have given a sufficient condition for a $T_{0}$ space to be well-filtered. We now give some new characterizations of well-filtered and sober spaces based on the results in the above section.

Proposition 3.1. Let $X$ be a $T_{0}$ space. Then the following two conditions are equivalent:

(1) $X$ is well-filtered.

(2) $\mathrm{WD}(X)=\mathcal{S}_{c}(X)$.

(3) $\operatorname{RD}(X)=\mathcal{S}_{c}(X)$.

Proof. $(1) \Rightarrow(2)$ : Use the mapping $i d_{X}: X \longrightarrow X$.

$(2) \Rightarrow(3)$ : By Proposition 2.6

$(3) \Rightarrow(1)$ : Suppose that $\mathcal{K} \subseteq \mathrm{K}(X)$ is filtered, $U \in \mathcal{O}(X)$, and $\bigcap \mathcal{K} \subseteq U$. If $K \nsubseteq U$ for each $K \in \mathcal{K}$, then by Lemma 2.1. the closed set $X \backslash U$ contains an irreducible closed subset $A$ that also meets all members of $\mathcal{K}$ and hence $A \in \operatorname{RD}(X)$. By $(2), A=\overline{\{x\}}$ for some $x \in X$. For each by $K \in \mathcal{K}$, since $K \cap A=K \cap \overline{\{x\}} \neq \emptyset$, we have $x \in K$. So $x \in \bigcap \mathcal{K} \subseteq U \subseteq X \backslash A$, a contradiction. Therefore, $K \subseteq U$ for some $K \in \mathcal{K}$.

Remark 3.2. The equivalence of (1) and (3) in Proposition 3.1 has been proved in [14] in a different way.

By Proposition 1.2 and Proposition 3.1, we get the following result.

Corollary 3.3. Every well-filtered space is a d-space.

Corollary 3.4. A retract of a well-filtered space is well-filtered. 
Proof. Suppose that $Y$ is a retract of a well-filtered space $X$. Then there are continuous mappings $f: X \longrightarrow$ $Y$ and $g: Y \longrightarrow X$ such that $f \circ g=i d_{Y}$. Let $B \in \operatorname{RD}(Y)$, then by Lemma 2.7 and Proposition 3.1, there exists a unique $x_{B} \in X$ such that $\overline{g(B)}=\overline{\left\{x_{B}\right\}}$. Therefore, $\bar{B}=\overline{f \circ g(B)}=\overline{f(\overline{g(B)})}=\overline{f\left(\overline{\left\{x_{B}\right\}}\right)}=\overline{\left\{f\left(x_{B}\right)\right\}}$. By Proposition 3.1, $Y$ is well-filtered.

By Theorem 2.10 and Proposition 3.1, we get the following well-known result.

Corollary 3.5. (4] [8]) Every locally compact well-filtered space is sober.

Example 3.6. Let $X$ be a countably infinite set and $X_{\text {cof }}$ the space equipped with the co-finite topology (the empty set and the complements of finite subsets of $X$ are open). Then

(a) $\mathcal{C}\left(X_{c o f}\right)=\{\emptyset, X\} \cup X^{(<\omega)}, X_{c o f}$ is $T_{1}$ and hence a strong $d$-space. (see Definition 3.18).

(b) $\mathrm{K}\left(X_{\text {cof }}\right)=2^{X} \backslash\{\emptyset\}$.

(c) $X_{\text {cof }}$ is locally compact and first countable.

(d) $\mathcal{S}_{c}\left(X_{\text {cof }}\right)=\mathcal{D}_{c}\left(X_{\text {cof }}\right)=\{\{x\}: x \in X\}$,

(e) $\operatorname{Irr}_{c}\left(X_{\text {cof }}\right)=\mathrm{WD}\left(X_{\text {cof }}\right)=\mathrm{RD}\left(X_{\text {cof }}\right)=\{X\} \cup\{\{x\}: x \in X\} \neq \mathcal{D}_{c}\left(X_{\text {cof }}\right)$. In fact, let $\mathcal{K}_{X}=\{X \backslash F$ : $\left.F \in X^{(<\omega)}\right\}$. Then $\mathcal{K}_{X} \in \mathrm{K}\left(X_{c o f}\right)$ is filtered and $X \in M\left(\mathcal{K}_{X}\right)$. For any $A \in \mathcal{C}\left(X_{c o f}\right)$, if $A \neq X$, then $A$ is finite and hence $A \notin M\left(\mathcal{K}_{X}\right)$ because $A \cap(X \backslash A)=\emptyset$. Thus $X \in \mathrm{RD}\left(X_{\text {cof }}\right)$, but $X \notin D_{c}\left(X_{\text {cof }}\right)$.

(f) $X_{\text {cof }}$ is not well-filtered (by Proposition 3.1).

Example 3.7. Let $L$ be the complete lattice constructed by Isbell 9]. Then by [16, Corollary 3.2] (or Corollary 3.17 below), $X=\Sigma L$ is a well-filtered space. Note that $\Sigma L$ is not sober. Thus by Proposition 3.1 . $\mathrm{WD}(X) \neq \operatorname{Irr}_{c}(X)$ and $\mathrm{RD}(X) \neq \operatorname{Irr}_{c}(X)$.

Example 3.8. Let $X$ be a uncountably infinite set and $X_{c o c}$ the space equipped with the co-countable topology (the empty set and the complements of countable subsets of $X$ are open). Then

(a) $\mathcal{C}\left(X_{c o c}\right)=\{\emptyset, X\} \cup X^{(\leqslant \omega)}, X_{c o c}$ is $T_{1}$ and hence a strong $d$-space.

(b) $\mathrm{K}\left(X_{c o c}\right)=X^{(<\omega)} \backslash\{\emptyset\}$ and int $K=\emptyset$ for all $K \in \mathrm{K}\left(X_{c o c}\right)$.

(c) $X_{c o c}$ is not locally compact and not first countable.

(d) $\operatorname{Irr}_{c}\left(X_{c o c}\right)=\{X\} \cup\{\{x\}: x \in X\}, \mathrm{WD}\left(X_{c o c}\right)=\operatorname{RD}\left(X_{c o c}\right)=\mathcal{D}_{c}\left(X_{c o c}\right)=\mathcal{S}_{c}\left(X_{c o c}\right)=\{\{x\}: x \in X\}$. Therefore, $\operatorname{Irr}_{c}\left(X_{c o c}\right) \neq \mathrm{WD}\left(X_{c o c}\right)$.

(e) $X_{c o c}$ is well-filtered (by Proposition 3.1), but it not sober.

Theorem 3.9. For any $T_{0}$ space $X$, the following conditions are equivalent:

(1) $X$ is well-filtered.

(2) For each $(A, K) \in \mathrm{WD}(X) \times \mathbf{u p}(X), \max (A) \neq \emptyset$ and $\downarrow(A \cap K) \in \mathcal{C}(X)$.

(3) For each $(A, K) \in \mathrm{RD}(X) \times \mathbf{u p}(X), \max (A) \neq \emptyset$ and $\downarrow(A \cap K) \in \mathcal{C}(X)$.

(4) For each $(A, K) \in \mathrm{WD}(X) \times \mathrm{K}(X)$, $\max (A) \neq \emptyset$ and $\downarrow(A \cap K) \in \mathcal{C}(X)$.

(5) For each $(A, K) \in \mathrm{RD}(X) \times \mathrm{K}(X)$, $\max (A) \neq \emptyset$ and $\downarrow(A \cap K) \in \mathcal{C}(X)$.

Proof. $(1) \Rightarrow(2)$ : Suppose that $X$ is well-filtered and $(A, K) \in \operatorname{Irr}_{c}(X) \times \mathbf{u p}(X)$. Then by Proposition 3.1 . there is $x \in X$ such that $A=\overline{\{x\}}$, and hence $\max (A)=\{x\} \neq \emptyset$. Now we show that $\downarrow(A \cap K)=\downarrow(\downarrow x \cap K)$ is closed. If $\downarrow(\downarrow x \cap K) \neq \emptyset$ (i.e., $\downarrow x \cap K \neq \emptyset)$, then $x \in K$ since $K$ is saturated (that is, $K$ is an upper set). It follows that $\downarrow(\downarrow x \cap K)=\downarrow x \in \mathcal{C}(X)$.

$(2) \Rightarrow(3)$ and $(4) \Rightarrow(5)$ : By Proposition 2.6

$(2) \Rightarrow(4)$ and $(3) \Rightarrow(5)$ : Trivial.

(5) $\Rightarrow(1)$ : Suppose that $\mathcal{K} \subseteq \mathrm{K}(X)$ is filtered, $U \in \mathcal{O}(X)$, and $\bigcap \mathcal{K} \subseteq U$. If $K \nsubseteq \subseteq$ for each $K \in \mathcal{K}$, then by Lemma 2.1. $X \backslash U$ contains a minimal irreducible closed subset $A$ that still meets all members of $\mathcal{K}$, and hence $A \in \operatorname{RD}(X)$. For any $\left\{K_{1}, K_{2}\right\} \subseteq \mathcal{K}$, we can find $K_{3} \in \mathcal{K}$ with $K_{3} \subseteq K_{1} \cap K_{2}$. It follows that $\downarrow\left(A \cap K_{1}\right) \in \mathcal{C}(X)$ and $\emptyset \neq A \cap K_{3} \subseteq \downarrow\left(A \cap K_{1}\right) \cap K_{2} \neq \emptyset$. By (5) and the minimality of $A$, we have $\downarrow\left(A \cap K_{1}\right)=A$ for all $K_{1} \in \mathcal{K}$. Select an $x \in \max (A)$. Then for each $K \in \mathcal{K}, x \in \downarrow(A \cap K)$, and consequently, there is $a_{k} \in A \cap K$ such that $x \leq a_{k}$. By the maximality of $x$ we have $x=a_{k}$. Therefore, $x \in K$ for all $K \in \mathcal{K}$, and so $x \in \bigcap \mathcal{K} \subseteq U \subseteq X \backslash A$, a contradiction. Thus $X$ is well-filtered. 
Note that if $X$ is a $d$-space, then by Lemma $1.3 \max (A) \neq \emptyset$ for every closed set $A$ of $X$. Therefore, by Theorem 3.9 , we obtain the following corollary.

Corollary 3.10. For a d-space $X$, the following conditions are equivalent:

(1) $X$ is well-filtered.

(2) For each $(A, K) \in \mathrm{WD}(X) \times \mathbf{u p}(X), \downarrow(A \cap K) \in \mathcal{C}(X)$.

(3) For each $(A, K) \in \mathrm{RD}(X) \times \mathbf{u p}(X) \downarrow \downarrow(A \cap K) \in \mathcal{C}(X)$.

(4) For each $(A, K) \in \mathrm{WD}(X) \times \mathrm{K}(X), \downarrow(A \cap K) \in \mathcal{C}(X)$.

(5) For each $(A, K) \in \mathrm{RD}(X) \times \mathrm{K}(X), \downarrow(A \cap K) \in \mathcal{C}(X)$.

Corollary 3.11. ([16]) Let $X$ be a d-space such that $\downarrow(A \cap K)$ is closed for all $A \in \mathcal{C}(X)$ and $K \in \mathrm{K}(X)$. Then $X$ is well-filtered.

Definition 3.12. Let $P$ be a poset equipped with a topology. The partial order is said to be upper semiclosed if each $\uparrow x$ is closed.

Definition 3.13. A topological space $X$ with a partially order is called upper semicompact, if $\uparrow x$ is compact for any $x \in X$, or equivalently, if $\uparrow x \cap A$ is compact for any $x \in X$ and $A \in \mathcal{C}(X)$. $X$ is called weakly upper semicompact if $\uparrow x \cap A$ is compact for any $x \in X$ and $A \in \operatorname{Irr}_{c}(X)$.

Lemma 3.14. (44) Let $X$ be a topological space with an upper semiclosed partial order. If $A$ is a compact subset of $X$, then $\downarrow$ A is Scott closed.

Lemma 3.15. Let $X$ be a $T_{0}$ space such that $\Sigma X$ is a d-space. For $A \in \mathcal{C}(X)$ and $K \in \mathrm{K}(\Sigma X)$, if $\downarrow(\uparrow x \cap A) \in \mathcal{C}(\Sigma X)$ for all $x \in X$, then $\downarrow(K \cap A)=\bigcup_{k \in K} \downarrow(\uparrow k \cap A) \in \mathcal{C}(\Sigma X)$.

Proof. Since $\Sigma X$ is a $d$-space, $X$ is a dcpo. Let $D \in \mathcal{D}(X)$ such that $D \subseteq \downarrow(K \cap A)$. If $\bigvee D \notin \downarrow(K \cap A)$, then for each $k \in K, \bigvee D \notin \downarrow(\uparrow k \cap A)$, and hence $\bigcap_{d \in D} \uparrow d \cap \downarrow(\uparrow k \cap A)=\emptyset$. For each $k \in K$, since $\Sigma X$ is a $d$-space and $\downarrow(\uparrow k \cap A) \in \sigma(X)$, by Proposition 1.2 there is a $d_{k} \in D$ such that $\uparrow d_{k} \cap \uparrow k \cap A=\emptyset$, and consequently, $k \in X \backslash \downarrow\left(\uparrow d_{k} \cap A\right)$ and $\downarrow\left(\uparrow d_{k} \cap A\right) \in \mathcal{C}(\Sigma X)$. By the compactness of $K$ in $\Sigma X$, there exists a finite subset $\left\{d_{k_{1}}, \ldots, d_{k_{n}}\right\} \subseteq D$ such that $K \subseteq \bigcup_{i=1}^{n}\left(X \backslash \downarrow\left(\uparrow d_{k_{i}} \cap A\right)\right)$. By the directness of $D$, there is a $d_{0}$ such that $\uparrow d_{0} \subseteq \bigcap_{i=1}^{n} \uparrow d_{k_{i}}$. It follows that $K \subseteq X \backslash \downarrow\left(\uparrow d_{o} \cap A\right)$, which contradicts $d_{o} \in \downarrow(K \cap A)$, hence $\bigvee D \in \downarrow(K \cap A)$.

By Corollary 3.10, Lemma 3.14 and Lemma 3.15, we get the following corollaries.

Corollary 3.16. For a dcpo $P$, if $(P, \lambda(P))$ is weakly upper semicompact, then $(P, \sigma(P))$ is well-filtered.

Corollary 3.17. ([16]) For a dcpo $P$, if $(P, \lambda(P))$ is upper semicompact (in particular, if $P$ is bounded complete), then $(P, \sigma(P))$ is well-filtered.

In order to reveal finer links between $d$-spaces and well-filtered spaces, we introduce another class of $T_{0}$ spaces.

Definition 3.18. A $T_{0}$ space $X$ is called a strong $d$-space if for any $D \in \mathcal{D}(X), x \in X$ and $U \in \mathcal{O}(X)$, $\bigcap_{d \in D} \uparrow d \cap \uparrow x \subseteq U$ implies $\uparrow d \cap \uparrow x \subseteq U$ for some $d \in D$.

Clearly, $X$ is a strong $d$-space iff for any $D \in \mathcal{D}(X), x \in X$ and $A \in \mathcal{C}(X)$, if $\uparrow d \cap \uparrow x \cap A \neq \emptyset$ for all $d \in D$, then $\bigcap_{d \in D}(\uparrow d \cap \uparrow x) \cap A \neq \emptyset$.

Every $T_{1}$ space is a strong $d$-space. Also it is easy to verify that every coherent well-filtered space is a strong $d$-space.

Proposition 3.19. For a $T_{0}$ space $X$, the following two conditions are equivalent:

(1) $X$ is a strong $d$-space.

(2) For any $D \in \mathcal{D}(X), \uparrow F \in \mathbf{F i n}(X)$ and $U \in \mathcal{O}(X), \bigcap_{d \in D} \uparrow d \cap \uparrow F \subseteq U$ implies $\uparrow d \cap \uparrow F \subseteq U$ for some $d \in D$. 
Proof. (1) $\Rightarrow(2)$ : Let $D \in \mathcal{D}, \uparrow F \in \mathbf{F i n}(X)$ and $U \in \mathcal{O}(X)$ such that $\bigcap_{d \in D} \uparrow d \cap \uparrow F \subseteq U$. Then for each $u \in F, \bigcap_{d \in D} \uparrow d \cap \uparrow u \subseteq U$, and hence $\uparrow d_{u} \cap \uparrow u \subseteq U$ for some $d_{u} \in D$. Since $F$ is finite and $D$ is a direct subset of $X$, there is a $d_{0} \in D$ such that $\uparrow d_{0} \subseteq \bigcap_{u \in F} \uparrow u$. It follows that $\uparrow d_{0} \cap \uparrow F \subseteq U$.

$(2) \Rightarrow(1)$ : Trivial.

Figure 1 shows certain relations of some spaces lying between $d$-spaces and $T_{2}$ spaces.

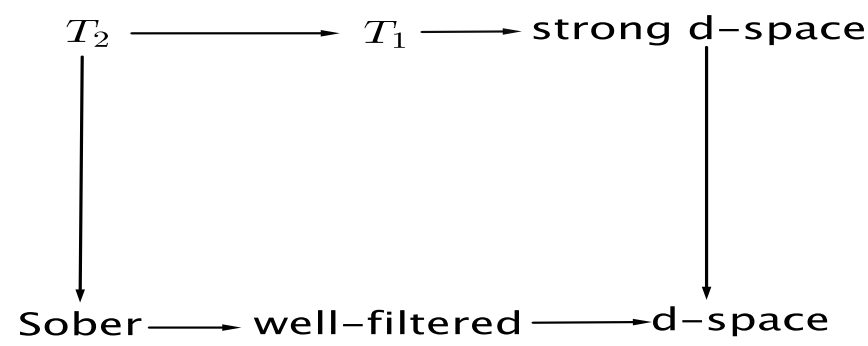

Figure 1: Relations of some spaces lying between $d$-spaces and $T_{2}$ spaces

Definition 3.20. Let $P$ be a dcpo. A subset $U \subseteq P$ is called strongly Scott open if (i) $U=\uparrow U$, and (ii) for any $D \in \mathcal{D}(P)$ and $x \in P, \bigcap_{d \in D} \uparrow d \cap \uparrow x \subseteq U$ (that is, $\uparrow \bigvee D \cap \uparrow x \subseteq U$ ) implies $\uparrow d \cap \uparrow x \subseteq U$ for some $d \in D$. Let $\sigma^{s}(P)$ denote the set of all strongly Scott open subsets of $P$.

Clearly, if $U, V \in \sigma^{s}(P)$, then $U \cap V \in \sigma^{s}(P)$. The topology generated by $\sigma^{s}(P)$ (as a base) is called the strong Scott topology on $P$ and denote it by $\sigma_{s}(P)$. The space $\left(P, \sigma_{s}(P)\right)$ is called the strong Scott space of $P$, and will be denote by $\Sigma_{s} P$.

For any $x, y$ in a poset $P$, if $\uparrow x \cap \downarrow y \neq \emptyset$, then $\downarrow(\uparrow x \cap \downarrow y)=\downarrow y$, and whence for any nonempty finite subset $F$ of $P, \downarrow(\uparrow x \cap \downarrow F)=\emptyset$ or $\downarrow(\uparrow x \cap \downarrow F)=\downarrow F_{x}$, where $F_{x}=\{u \in F: \uparrow x \cap \downarrow u \neq \emptyset\}$. Now we check $P \backslash \downarrow F \in \sigma^{s}(P)$. For any $D \in \mathcal{D}(P)$ and $x \in X$, if $\bigcap_{d \in D} \uparrow d \cap \uparrow x \subseteq P \backslash \downarrow F$, then $\bigcap_{d \in D} \uparrow d \cap \uparrow x \cap \downarrow F=\emptyset$, and whence $\bigcap_{d \in D} \uparrow d \cap \downarrow(\uparrow x \cap \downarrow F)=\emptyset$, or equivalently, $\bigcap_{d \in D} \uparrow d \subseteq P \backslash \downarrow(\uparrow x \cap \downarrow F) \in v(P) \subseteq \sigma(P)$. Therefore, $\uparrow d \subseteq P \backslash \downarrow(\uparrow x \cap \downarrow F)$ for some $d \in D$, and so $\uparrow d \cap \uparrow x \subseteq P \backslash \downarrow F$. Thus we have the following relations:

$$
v(P) \subseteq \sigma_{s}(P) \subseteq \sigma(P)
$$

Therefore, if $(P, v(P))$ is not a strong $d$-space, then any space $(P, \tau)$ with $v(P) \subseteq \tau \subseteq \alpha(P)$ is not a strong $d$-space. In particular, $\Sigma_{s} P$ and $\Sigma P$ are not strong $d$-spaces. For two topologies $\tau_{1}$ and $\tau_{2}$ on $P$ with $v(P) \subseteq \tau_{1} \subseteq \tau_{2}$, if $\left(P, \tau_{2}\right)$ is a strong $d$-space, then $\left(P, \tau_{1}\right)$ is also a strong $d$-space.

Remark 3.21. (1) If a dcpo $P$ is a sup semilattice, then $\sigma_{s}(P)=\sigma(P)$. In this case, $(P, \sigma(P))$ is a strong $d$-space.

(2) For a dcpo $P$, if $\sigma_{s}(P)=\sigma^{s}(P)$, then $\Sigma_{s} P$ is a strong $d$-space.

Proposition 3.22. For a $T_{0}$ space $X$, consider the following two conditions:

(1) $X$ is a strong d-space.

(2) $X$ is a d-space, and $\mathcal{O}(X) \subseteq \sigma_{s}(X)$.

Then $(1) \Rightarrow(2)$, and the two conditions are equivalent if $X$ (with the specialization order) is a sup semilattice.

Proof. $(1) \Rightarrow(2)$ : Let $D \in \mathcal{D}(X)$ and $U \in \mathcal{O}(X)$ with $\bigcap_{d \in D} \uparrow d \subseteq U$. Then for any $c \in D$,

$$
\bigcap_{d \in D} \uparrow d \cap \uparrow c=\bigcap_{d \in D} \uparrow d \subseteq U
$$

and hence $\uparrow d \cap \uparrow c \subseteq U$ for some $d \in D$. Take one $e \in D$ with $d \leq e$ and $e \leq c$. Then $\uparrow e \subseteq \uparrow d \cap \uparrow c \subseteq U$. By Proposition 1.2, $X$ is a $d$-space. Now we prove that $\mathcal{O}(X) \subseteq \sigma_{s}(X)$. Suppose $U \in \mathcal{O}(X), x \in X$ and 
$D \in \mathcal{D}(X)$ such that $\bigcap_{d \in D} \uparrow d \cap \uparrow x \subseteq U$. Since $X$ is a strong $d$-space, $\uparrow d \cap \uparrow x \subseteq U$ for some $d \in D$. Thus $U$ is strongly Scott open.

$(2) \Rightarrow(1)$ : Suppose that $X$ is a sup semilattice. For any $D \in \mathcal{D}, x \in X$ and $U \in \mathcal{O}(X)$, if $\bigcap_{d \in D} \uparrow d \cap \uparrow x \subseteq$ $U$, then $\bigcap_{d \in D} \uparrow(d \vee x) \subseteq U$ and $\{d \vee x: d \in D\} \in \mathcal{D}(X)$. By Proposition $1.2 \uparrow d \cap \uparrow x=\uparrow d \vee x \subseteq U$ for some $d \in D$. Thus $X$ is a strong $d$-space.

Definition 3.23. A poset $P$ is said to have property $D$ if for any nonempty subset $\left\{x_{i}: i \in I\right\} \subseteq P$ that has a lower bound (that is $\left.\bigcap_{i \in I} \downarrow x_{i} \neq \emptyset\right), \bigcap_{i \in I} \downarrow x_{i} \in \operatorname{Id}(P)$.

Clearly, every bounded complete poset has property $D$. For a dcpo $P, P$ satisfies property $D$ iff every nonempty subset $\left\{x_{i}: i \in I\right\} \subseteq P$ that has a lower bound has the greatest lower bound (that is, $\bigcap_{i \in I} \downarrow x_{i}$ is a principal ideal of $P$ ).

Lemma 3.24. For a poset $P$, the following two conditions are equivalent:

(1) $(P, v(P))$ is a strong d-space.

(2) $P$ is a dcpo, and for any $\left\{F_{i}: i \in I\right\} \subseteq P^{(<\omega)}$ and $x \in P, \downarrow\left(\uparrow x \cap \bigcap_{i \in I} \downarrow F_{i}\right) \in \mathcal{C}(\Sigma P)$.

Proof. $(1) \Rightarrow(2)$ : Suppose that $(P, v(P))$ is a strong $d$-space. Then $(P, v(P))$ is a $d$-space, and hence $P$ is a dcpo. For $\left\{F_{i}: i \in I\right\} \subseteq P^{(<\omega)}$ and $x \in P$, we show that $\downarrow \bigcap_{i \in I}\left(\uparrow x \cap \downarrow F_{i}\right) \in \mathcal{C}(\Sigma P)$. For any $D \in \mathcal{D}(P)$ with $D \subseteq \downarrow\left(\uparrow x \cap \bigcap_{i \in I} \downarrow F_{i}\right)$, if $\bigvee D \notin \downarrow\left(\uparrow x \cap \bigcap_{i \in I} \downarrow F_{i}\right)$, then $\uparrow \bigvee D \cap \uparrow x \cap \bigcap_{i \in I} \downarrow F_{i}=\bigcap_{d \in D} \uparrow d \cap \uparrow x \cap \bigcap_{i \in I} \downarrow F_{i}=\emptyset$, and whence $\bigcap_{d \in D} \uparrow d \cap \uparrow x \subseteq P \backslash \bigcap_{i \in I} \downarrow F_{i} \in v(P)$. Since $(P, v(P))$ is a strong $d$-space, there is $d \in D$ such that $\uparrow d \cap \uparrow x \subseteq P \backslash \bigcap_{i \in I} \downarrow F_{i}$, which is a contradiction with $d \in \downarrow\left(\uparrow x \cap \bigcap_{i \in I} \downarrow F_{i}\right)$. Therefore, $\bigvee D \in \downarrow\left(\uparrow x \cap \bigcap_{i \in I} \downarrow F_{i}\right)$. Thus $\downarrow\left(\uparrow x \cap \bigcap_{i \in I} \downarrow F_{i}\right) \in \mathcal{C}(\Sigma P)$.

$(2) \Rightarrow(1)$ : For any $D \in \mathcal{D}(P), x \in P$ and $U \in v(P)$ such that $\bigcap_{d \in D} \uparrow d \cap \uparrow x \subseteq U$, if $U=P$, then $\uparrow d \cap \uparrow x \subseteq U$ for all $d \in D$; if $U$ is a proper $v$-open subset of $P$, then there is a family $\left\{F_{i}: i \in I\right\} \subseteq P^{(<\omega)}$ such that $U=P \backslash \bigcap_{i \in I} \downarrow F_{i}$. Therefore, $\uparrow \bigvee D=\bigcap_{d \in D} \uparrow d \subseteq P \backslash \downarrow\left(\uparrow x \cap \bigcap_{i \in I} \downarrow F_{i}\right) \in \sigma(P)$ by condition (2). It follows that $\uparrow d \subseteq P \backslash \downarrow\left(\uparrow x \cap \bigcap_{i \in I} \downarrow F_{i}\right)$ for some $d \in D$, and whence $\uparrow d \cap \uparrow x \subseteq P \backslash \bigcap_{i \in I} \downarrow F_{i}$, proving that $(P, v(P))$ is a strong $d$-space.

Similarly, we have the following result.

Lemma 3.25. For a poset $P$, the following two conditions are equivalent:

(1) $\Sigma P$ is a strong d-space.

(2) $P$ is a dcpo, and for any $A \in \mathcal{C}(\Sigma P)$ and $x \in P, \downarrow(\uparrow x \cap A) \in \mathcal{C}(\Sigma P)$.

Corollary 3.26. For a dcpo $P$ satisfying property $D$ (in particular, $P$ is a complete semilattice), $(P, v(P)$ ) is a strong d-space.

Proof. For any $\left\{F_{i}: i \in I\right\} \subseteq P^{(<\omega)}$ and $x \in P$, we show that $\downarrow\left(\uparrow x \cap \bigcap_{i \in I} \downarrow F_{i}\right)=\bigcap_{i \in I} \downarrow\left(\uparrow x \cap \downarrow F_{i}\right)$. Obviously, $\downarrow\left(\uparrow x \cap \bigcap_{i \in I} \downarrow F_{i}\right) \subseteq \bigcap_{i \in I} \downarrow\left(\uparrow x \cap \downarrow F_{i}\right)$. Conversely, if $y \in \bigcap_{i \in I} \downarrow\left(\uparrow x \cap \downarrow F_{i}\right)$, then for each $i \in I$, there exists $u_{i} \in \uparrow x \cap \downarrow F_{i}$ with $y \leq u_{i}$, and hence there is $t_{i} \in F_{i}$ such that $u_{i} \leq t_{i}$. It follows that $x, y \in \bigcap_{i \in I} \downarrow t_{i}$. Since $P$ satisfies property $D$, there is a $z \in \bigcap_{i \in I} \downarrow t_{i} \subseteq \bigcap_{i \in I} \downarrow F_{i}$ such that $y \leq z$ and $x \leq z$, and whence $z \in \uparrow x \cap \bigcap_{i \in I} \downarrow F_{i}$ and $y \in \downarrow\left(\uparrow x \cap \bigcap_{i \in I} \downarrow F_{i}\right)$. Thus $\downarrow\left(\uparrow x \cap \bigcap_{i \in I} \downarrow F_{i}\right)=\bigcap_{i \in I} \downarrow\left(\uparrow x \cap \downarrow F_{i}\right)$. For any $s, t \in P$, if $\uparrow s \cap \downarrow t \neq \emptyset$, then $t \in \uparrow s \cap \downarrow t$, and hence $\downarrow(\uparrow s \cap \downarrow t)=\downarrow t$. Therefore, for each $i \in I, \downarrow\left(\uparrow x \cap \downarrow F_{i}\right)=\bigcup_{t \in F_{i}} \downarrow(\uparrow x \cap \downarrow t)=\downarrow F_{i}^{x}$, where $F_{i}^{x}=\left\{t \in F_{i}: \uparrow x \cap \downarrow t \neq \emptyset\right\}$. It follows that $\downarrow\left(\uparrow x \cap \bigcap_{i \in I} \downarrow F_{i}\right)=\bigcap_{i \in I} \downarrow\left(\uparrow x \cap \downarrow F_{i}\right) \in \mathcal{C}((P, v(P))) \subseteq \mathcal{C}(\Sigma P)$. By lemma 3.24. $(P, v(P))$ is a strong $d$-space.

Remark 3.27. For a dcpo $P$, consider the following three conditions:

(1) $P$ has property $D$.

(2) For any $\left\{F_{i}: i \in I\right\} \subseteq P^{(<\omega)}$ and $x \in P, \downarrow\left(\bigcap_{i \in I}\left(\uparrow x \cap \downarrow F_{i}\right)\right)=\bigcap_{i \in I} \downarrow\left(\uparrow x \cap \downarrow F_{i}\right)$ (note that $\bigcap_{i \in I} \downarrow\left(\uparrow x \cap \downarrow F_{i}\right)$ is always $v$-closed).

(3) $(P, v(P))$ is a $d$-space. 
Then by Lemma 3.24 and the proof of Corollary 3.26 , we have $(1) \Rightarrow(2) \Rightarrow(3)$.

Proposition 3.28. If $X$ is a d-space and $\downarrow(\uparrow x \cap A) \in \mathcal{C}(X)$ for all $x \in X$ and $A \in \mathcal{C}(X)$, then $X$ is a strong d-space.

Proof. Suppose that $D \in \mathcal{D}, x \in X$ and $U \in \mathcal{O}(X)$ such that $\bigcap_{d \in D} \uparrow d \cap \uparrow x \subseteq U$. Let $A=X \backslash U$. Then $A \in \mathcal{C}(X)$. If for any $d \in D, \uparrow d \cap \uparrow x \nsubseteq U$, then $\uparrow d \cap \downarrow(\uparrow x \cap A) \neq \emptyset$. Since $X$ is a $d$-space and $\downarrow(\uparrow x \cap A) \in \mathcal{C}(X)$, by Proposition 1.2, we have $\bigcap_{d \in D} \uparrow d \cap \downarrow(\uparrow x \cap A) \neq \emptyset$, and hence $\bigcap_{d \in D} \uparrow d \cap \uparrow x \cap A \neq \emptyset$, a contradiction. Thus $\uparrow d \cap \uparrow x \subseteq U$ for some $d \in D$.

By Theorem 3.9. Corollary 3.10, Lemma 3.14 and Proposition 3.28, we get the following corollaries.

Corollary 3.29. For a $T_{0}$ space $X$, if for each $B \in \mathrm{WD}(X)$ and $(A, K) \in \mathcal{C}(X) \times \mathrm{K}(X)$, $\max (B) \neq \emptyset$ and $\downarrow(A \cap K) \in \mathcal{C}(X)$, then $X$ is a well-filtered strong d-space.

Corollary 3.30. For a $T_{0}$ space $X$, if for each $B \in \mathrm{RD}(X)$ and $(A, K) \in \mathcal{C}(X) \times \mathrm{K}(X)$, max $(B) \neq \emptyset$ and $\downarrow(A \cap K) \in \mathcal{C}(X)$, then $X$ is a well-filtered strong d-space.

Corollary 3.31. Let $X$ be a d-space such that $\downarrow(A \cap K)$ is closed for all $A \in \mathcal{C}(X)$ and $K \in \mathrm{K}(X)$. Then $X$ is a well-filtered strong d-space.

Corollary 3.32. For a dcpo $P$, if $(P, \lambda(P))$ is upper semicompact, then $(P, \sigma(P))$ is a strong d-space.

The following example shows that for a dcpo $P,(P, v(P))$ and $(P, \sigma(P))$ need not be strong $d$-spaces, although they are always $d$-spaces.

Example 3.33. (Johnstone space) Let $\mathbb{J}=\mathbb{N} \times(\mathbb{N} \cup\{\omega\})$ with ordering defined by $(j, k) \leq(m, n)$ iff $j=m$ and $k \leq n$, or $n=\omega$ and $k \leq m$ (see [11]). Then $\mathbb{J}$ is a dcpo, and hence the Johnstone space $\Sigma \mathbb{J}$ is a $d$-space. However, $\Sigma \mathbb{J}$ is not well-filtered (see [5, Exercise 8.3.9]), and hence non-sober. Clearly, $\bigcap_{n \in \mathbb{N}}(\uparrow(1, n) \cap \uparrow(2,1))=\emptyset$, but $\uparrow(1, n) \cap \uparrow(2,1)=\{(m, \omega): n \leq m\} \neq \emptyset$ for all $n$. Hence $(\mathbb{J}, v(\mathbb{J}))$ and $\Sigma \mathbb{J}$ are not strong $d$-spaces.

The following example shows that even for a continuous dcpo $P$, if $\Sigma P$ is not coherent, $\Sigma P$ and $\Sigma_{s} P$ may not be strong $d$-spaces.

Example 3.34. Let $C=\left\{a_{1}, a_{2}, \ldots, a_{n}, \ldots\right\} \cup\left\{\omega_{0}\right\}$ and $P=C \cup\{b\} \cup\left\{\omega_{1}, \ldots, \omega_{n}, \ldots\right\}$ with the order generated by

(a) $a_{1}<a_{2}<\ldots<a_{n}<a_{n+1}<\ldots$;

(b) $a_{n}<\omega_{0}$ for all $n \in \mathbb{N}$;

(c) $b<\omega_{n}$ and $a_{m}<\omega_{n}$ for all $n, m \in \mathbb{N}$ with $m \leq n$.

Then $P$ is a dcpo and $D \in \mathcal{D}(P)$ iff $D \subseteq C$ or $D$ has a largest element (that is $\downarrow D$ is a principal ideal of $P$ ), and hence $x \ll x$ for all $x \in P \backslash\left\{\omega_{0}\right\}$. Therefore, $P$ is a continuous dcpo and $\Sigma P$ is sober. $\uparrow a_{1}, \uparrow b \in \mathrm{K}(\Sigma)$, but $\uparrow a_{1} \cap \uparrow b=\left\{\omega_{1}, \omega_{2}, \ldots, \omega_{n}, \ldots\right\}$ is not Scott compact (note $\left\{\omega_{n}\right\} \in \sigma(P)$ for all $n \in \mathbb{N}$ ). Thus $\Sigma P$ is not coherent. For any $m \in \mathbb{N}, \bigcap_{n \in \mathbb{N}} \uparrow a_{n} \cap \uparrow b=\emptyset \subseteq\left\{\omega_{m}\right\} \in \sigma(P)$, but $\uparrow a_{n} \cap \uparrow b=\left\{\omega_{n}, \omega_{n+1}, \ldots\right\} \nsubseteq\left\{\omega_{m}\right\}$ for any $n \in \mathbb{N}$. Thus $\Sigma P$ is not a strong $d$-space. Since $\bigcap_{n \in \mathbb{N}} \uparrow a_{n} \cap \uparrow b=\emptyset$, but $\uparrow a_{n} \cap \uparrow b=\left\{\omega_{n}, \omega_{n+1}, \ldots\right\} \neq \emptyset$, $(P, v(P))$ is not a strong $d$-space, and hence $\Sigma_{s} P$ is not a strong $d$-space because $v(P) \subseteq \sigma_{s}(P)$.

Remark 3.35. Let $P$ be the continuous domain in Example 3.34 . Then $\downarrow(\uparrow b \cap P)=P \backslash\left\{\omega_{0}\right\}$ is not Scott closed since $\left\{\omega_{0}\right\} \notin \sigma(P)$ (or equivalently, $\omega_{0}$ is not a compact element of $P$ ). So as a sufficient condition for a $d$-space to be well-filtered, the condition that $\downarrow(A \cap K)$ is closed for all $A \in \mathcal{C}(X)$ and $K \in \mathrm{K}(X)$ seems a little too strong (see Corollary 3.10, Corollary 3.11 and Corollary 3.31.

Lemma 3.36. ([7]) For a $T_{0}$ space $X$, the following conditions are equivalent:

(1) $X$ is sober.

(2) For any $\mathcal{A} \subseteq \operatorname{Irr}\left(P_{S}(X)\right)$ and $U \in \mathcal{O}(X), \bigcap \mathcal{A} \subseteq U$ implies $K \subseteq U$ for some $K \in U$. 


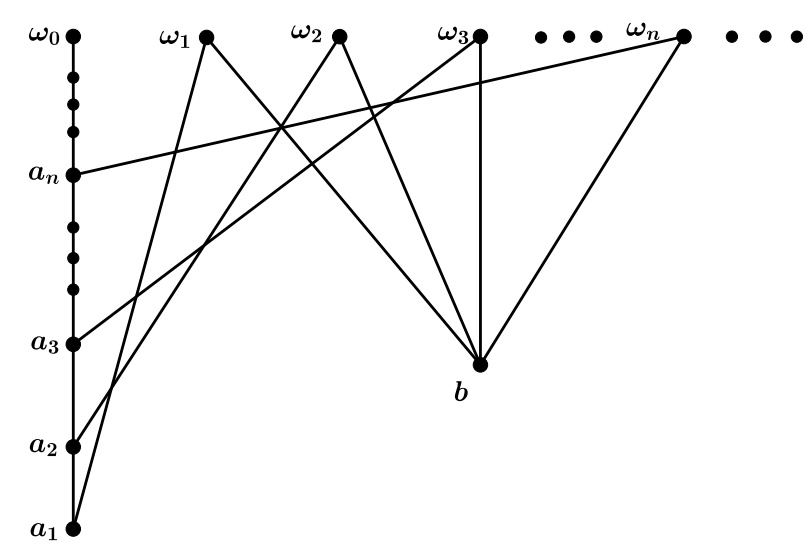

Figure 2: The Poset $P$

(3) $P_{S}(X)$ is sober.

Remark 3.37. By Remark 1.8 and Lemma 3.36, we have that $X$ is sober iff for any $\mathcal{A} \subseteq \operatorname{Irr}_{c}\left(P_{S}(X)\right)$ and $U \in \mathcal{O}(X), \bigcap \mathcal{A} \subseteq U$ implies $K \subseteq U$ for some $K \in \mathcal{A}$.

For a $T_{0}$ space and $A \subseteq X$, define $\Psi_{\mathbf{u p}(X)}(A)=\{K \in \mathbf{u p}(X): K \cap A \neq \emptyset\}$ and $\Psi_{\mathrm{K}(X)}(A)=\{K \in$ $\mathrm{K}(X): K \cap A \neq \emptyset\}$.

The following theorem provides a new characterization of sober spaces similar to that for well-filtered spaces given in Theorem 3.9 .

Theorem 3.38. The following conditions are equivalent for a $T_{0}$ space $X$ :

(1) $X$ is sober.

(2) For any $(A, K) \in \operatorname{Irr}_{c}(X) \times \mathbf{u p}(X), \Psi_{\mathbf{u p}(X)}(A) \in \operatorname{Filt}(\mathbf{u p}(X)), \max (A) \neq \emptyset$ and $\downarrow(A \cap K) \in \mathcal{C}(X)$.

(3) For any $(A, K) \in \operatorname{Irr}_{c}(X) \times \mathrm{K}(X), \Psi_{\mathrm{K}(X)}(A) \in \operatorname{Filt}(\mathrm{K}(X)), \max (A) \neq \emptyset$ and $\downarrow(A \cap K) \in \mathcal{C}(X)$.

Proof. We only prove the equivalence of (1) and (3). The proof of the equivalence of (1) and (3) is similar.

$(1) \Rightarrow(3)$ : Suppose that $X$ is sober and $(A, K) \in \operatorname{Irr}_{c}(X) \times \mathrm{K}(X)$. Then there is an $x \in X$ such that $A=\overline{\{x\}}$, and hence $\max (A)=\{x\} \neq \emptyset$. Clearly, $\Psi_{\mathrm{K}(X)}(A)=\{K \in \mathrm{K}(X): K \cap \downarrow x \neq \emptyset\}=\uparrow_{\mathrm{K}(X)} \uparrow_{x}$ is a principal filter of $\mathrm{K}(X)$. Now we show that $\downarrow(A \cap K)$ is a closed subset of $X$. Obviously, if $\downarrow x \cap K=\emptyset$, then $\downarrow(A \cap K)=\downarrow(\downarrow x \cap K)=\emptyset$; if $\downarrow x \cap K \neq \emptyset$ (in this case $x \in K$ since $K$ is an upper set), then $\downarrow(A \cap K)=\downarrow(\downarrow x \cap K)=\downarrow x$. Thus $\downarrow(A \cap K)=\downarrow x \in \mathcal{C}(X)$.

$(3) \Rightarrow(1)$ : Suppose $\mathcal{A} \subseteq \operatorname{Irr}\left(P_{S}(X)\right)$ and $U \in \mathcal{O}(X)$ such that $\bigcap \mathcal{A} \subseteq U$. If $K \nsubseteq U$ for all $K \in U$, then by Lemma 2.1 $X \backslash U$ contains a minimal irreducible closed subset $A$ that still meets all members of $\mathcal{A}$. For any $\left\{K_{1}, K_{2}\right\} \subseteq \mathcal{A}$, since $\Psi_{\mathrm{K}(X)}(A) \in \mathrm{Filt}(\mathrm{K}(X))$, there is a $K_{3} \in \Psi_{\mathrm{K}(X)}(A)$ with $K_{3} \subseteq K_{1} \cap K_{2}$. It follows that $\emptyset \neq A \cap K_{3} \subseteq \downarrow\left(A \cap K_{1}\right) \cap K_{2} \neq \emptyset$. By (3) and the minimality of $A$, we have $\downarrow\left(A \cap K_{1}\right)=A$ for all $K_{1} \in \mathcal{A}$. Select an $x \in \max (A)$. Then for each $K \in \mathcal{A}, x \in \downarrow(A \cap K)$, and consequently, there is $a_{k} \in A \cap K$ such that $x \leq a_{k}$. By the maximality of $x$, we have $x=a_{k}$. Therefore, $x \in K$ for all $K \in \mathcal{A}$, and whence $x \in \bigcap \mathcal{A} \subseteq U \subseteq X \backslash A$, a contradiction. By Lemma 3.36, $X$ is sober.

By Lemma 1.3 and Theorem 3.38, we get the following corollary.

Corollary 3.39. For a d-space $X$, the following conditions are equivalent:

(1) $X$ is sober.

(2) For each $(A, K) \in \operatorname{Irr}_{c}(X) \times \mathbf{u p}(X), \Psi_{\mathbf{u p}(X)}(A) \in \operatorname{Filt}(\mathbf{u p}(X))$ and $\downarrow(A \cap K) \in \mathcal{C}(X)$.

(3) For each $(A, K) \in \operatorname{Irr}_{c}(X) \times \mathrm{K}(X), \Psi_{\mathrm{K}(X)}(A) \in \operatorname{Filt}(\mathrm{K}(X))$ and $\downarrow(A \cap K) \in \mathcal{C}(X)$. 


\section{Conclusion}

In this paper, based on topological Rudin's Lemma, we instigated two new classes of subsets lying between the classes of all closures of directed subsets and that of irreducible closed sets. Using such subsets, we obtained some new characterizations of sober spaces and well-filtered spaces, which improve and generalize the related results of Xi and Lawson [16.

Our study also leads to the definition of a new class of spaces - strong $d$-spaces and a new topology - the strong Scott topology which may deserve further investigation.

We now close our paper with some problems.

Problem 4.1. Does $\operatorname{RD}(X)=\mathrm{WD}(X)$ hold for every $T_{0}$ space $X$ ?

Problem 4.2. Does $\operatorname{Irr}_{c}(X)=\mathrm{WD}(X)$ hold for every core compact $T_{0}$ space $X$ ?

Problem 4.3. Are the following conditions equivalent for any $T_{0}$ space $X$ ?

(a) $X$ is sober.

(b) For each $(A, K) \in \operatorname{Irr}_{c}(X) \times \mathbf{u p}(X), \max (A) \neq \emptyset$ and $\downarrow(A \cap K) \in \mathcal{C}(X)$.

(c) For each $(A, K) \in \operatorname{Irr}_{c}(X) \times \mathrm{K}(X), \max (A) \neq \emptyset$ and $\downarrow(A \cap K) \in \mathcal{C}(X)$.

Problem 4.4. Are the following conditions equivalent for any $d$-space $X$ ?

(a) $X$ is sober.

(b) For each $(A, K) \in \operatorname{Irr}_{c}(X) \times \mathbf{u p}(X), \downarrow(A \cap K) \in \mathcal{C}(X)$.

(c) For each $(A, K) \in \operatorname{Irr}_{c}(X) \times \mathrm{K}(X), \downarrow(A \cap K) \in \mathcal{C}(X)$.

\section{References}

\section{References}

[1] M. Erné, The strength of prime ideal separation, sobriety, and compactness theorems. Topol. Appl. 241 (2018) $263-290$.

[2] M. Erné, Categories of Locally Hypercompact Spaces and Quasicontinuous Posets, Applied Categorical Structures. 26 (2018) 823-854.

[3] M. Escard́o, J. Lawson, A. Simpson, Comparing Cartesian closed categories of (core) compactly generated spaces, Topol. Appl. 143 (2004) 105C145.

[4] G. Gierz, K. Hofmann, K. Keimel, J. Lawson, M. Mislove, D. Scott, Continuous Lattices and Domains, Encycl. Math. Appl., vol. 93, Cambridge University Press, 2003.

[5] J. Goubault-Larrecq, Non-Hausdorff topology and Domain Theory, New Mathematical Monographs, vol. 22, Cambridge University Press, 2013.

[6] R. Heckmann, An upper power domain construction in terms of strongly compact sets, in: Lecture Notes in Computer Science, vol. 598, Springer, Berlin Heidelberg New York, 1992, pp. 272-293.

[7] R. Heckmann, K. Keimel, Quasicontinuous domains and the Smyth powerdomain, Electronic Notes in Theor. Comp. Sci. 298 (2013) 215-232.

[8] H. Kou, $U_{k}$-admitting dcpos need not be sober, in: Domains and Processes, Semantic Structure on Domain Theory, vol. 1, Kluwer, 2001, pp. 41-50.

[9] J. Isbell, Completion of a construction of Johnstone, Proc. Amer. Math. Soci. 85 (1982) 333-334.

[10] X. Jia, A. Jung, A note on coherence of dcpos, Topol. Appl. 209 (2016) 235-238.

[11] P. Johnstone, Scott is not always sober, in: Continuous Lattices, Lecture Notes in Math., vol. 871, Springer-Verlag, 1981, pp. 282-283.

[12] M. Rudin, Directed sets which converge, in: General Topology and Modern Analysis, University of California, Riverside, 1980, Academic Press, 1981, pp. 305C307.

[13] A. Schalk, Algebras for Generalized Power Constructions, PhD Thesis, Technische Hochschule Darmstadt, 1993.

[14] C. Shen, X. Xi, X. Xu, D. Zhao, On well-filtered reflections of $T_{0}$ spaces, Topol. Appl. 267 (2019) 106869. DOI: 10.1016/j.topol.2019.106869.

[15] U. Wyler, Dedekind complete posets and Scott topologies, in: Lecture Notes in Mathematics, vol. 871, 1981, pp. 384-389.

[16] X. Xi, J. Lawson, On well-filtered spaces and ordered sets, Topol. Appl. 228 (2017) 139-144.

[17] D. Zhao, W. Ho, On topologies defined by irreducible sets, Journal of Logical and Algebraic Methods in Programmin. 84(1) (2015) 185-195 